\title{
High prevalence of occult left heart disease in scleroderma-pulmonary hypertension
}

\author{
Benjamin D. Fox ${ }^{1,2}$, Avi Shimony1, David Langleben', Andrew Hirsch1, \\ Lawrence Rudski ${ }^{1}$, Robert Schlesinger ${ }^{1}$, Mark J. Eisenberg ${ }^{1}$, Dominique Joyal ${ }^{1}$, \\ Marie Hudson ${ }^{1}$, Kim Boutet $^{3}$, Alexandrina Serban ${ }^{3}$, Ariel Masetto ${ }^{4}$ and \\ Murray Baron ${ }^{1}$
}

Affiliations: ${ }^{1}$ Center for Pulmonary Vascular Disease, Divisions of Cardiology, Respirology and Rheumatology, and Lady Davis Institute for Medical Research, Jewish General Hospital, McGill University, Montreal, QC, ${ }^{3}$ Division of Respirology, Hopital du Sacre Coeur, University of Montreal, Montreal, QC, and ${ }^{4}$ Division of Rheumatology, Centre Hospitalier Universite de Sherbrooke, Sherbrooke, QC, Canada. ${ }^{2}$ Pulmonary Institute, Rabin Medical Center, Petach Tikva, Israel.

Correspondence: D. Langleben, Room E-206, Jewish General Hospital, 3755 Cote Ste Catherine, Montreal, Quebec, Canada H3T 1E2. E-mail: david.langlebendamcgill.ca

ABSTRACT Our study aimed to determine the prevalence of occult left-heart disease in patients with scleroderma and pulmonary hypertension. In patients with pulmonary hypertension (mean pulmonary artery pressure (mean PAP) $\geqslant 25 \mathrm{mmHg}$ ), differentiation between pre- and post-capillary pulmonary hypertension has been made according to pulmonary artery wedge pressure (PAWP) less than or more than $15 \mathrm{mmHg}$, respectively.

We performed a retrospective chart review of 107 scleroderma patients. All patients with suspected pulmonary hypertension had routine right or left heart catheterisation with left ventricular end-diastolic pressure (LVEDP) measurement pre-/post-fluid challenge. We extracted demographic, haemodynamic and echocardiographic data. Patients were classified into one of four groups: haemodynamically normal (mean $\mathrm{PAP}<25 \mathrm{mmHg}$ ); pulmonary venous hypertension $(\mathrm{PVH})$ (mean $\mathrm{PAP} \geqslant 25 \mathrm{mmHg}, \mathrm{PAWP}>15 \mathrm{mmHg}$ ); occult PVH (mean PAP $\geqslant 25 \mathrm{mmHg}$, PAWP $\leqslant 15 \mathrm{mmHg}$, LVEDP $>15 \mathrm{mmHg}$ before or after fluid challenge); and pulmonary arterial hypertension (PAH) (mean PAP $\geqslant 25 \mathrm{mmHg}, \mathrm{PAWP} \leqslant 15 \mathrm{mmHg}$ and LVEDP $\leqslant 15 \mathrm{mmHg}$ before or after fluid challenge).

53 out of 107 patients had pulmonary hypertension. Based on the PAWP-based definition, 29 out of 53 had PAH and 24 out of 53 had PVH. After considering the resting and post-fluid-challenge LVEDP, 11 PAH patients were reclassified as occult PVH. The occult PVH group was haemodynamically, echocardiographically and demographically closer to the PVH group than the PAH group.

$\mathrm{PVH}$ had high prevalence in our scleroderma-pulmonary hypertension population. Distinguishing $\mathrm{PAH}$ from PVH with only PAWP may result in some PVH patients being misclassified as having PAH.

@ERSpublications

Left heart catheterisation with saline infusion helps in the diagnosis of suspected $\mathrm{PH}$ in scleroderma http://ow.ly/mxuXE

For editorial comments see page 888 .

Received: June 082012 | Accepted after revision: Dec 092012 | First published online: Dec 202012

Support statement: B.D. Fox was supported by the William Pencer Family Endowment at the Jewish General Hospital, and the American Fellowships Program for Physicians in Israel. This study was supported in part by the Bank of Montreal Center for the Study of Heart Disease in Women at the Jewish General Hospital, and by the Dimitrios Banousis Foundation.

Conflict of interest: Disclosures can be found alongside the online version of this article at www.erj.ersjournals.com 


\section{Introduction}

The association between scleroderma and pulmonary hypertension (PH) is well established [1]. It may be caused by pre-capillary microvascular narrowing resulting in pulmonary arterial hypertension (PAH) or pulmonary parenchymal disease, veno-occlusive disease, post-capillary pulmonary venous hypertension (PVH) from left heart dysfunction or combinations of these abnormalities [2]. The pulmonary vasodilator drugs approved for PAH may have deleterious effects or be ineffective if administered to patients with $\mathrm{PVH}$ or lung disease. Right heart catheterisation is essential for accurate diagnosis of all patients with suspected $\mathrm{PH}$. Typically, differentiation between $\mathrm{PAH}$ and $\mathrm{PVH}$ is based on the pulmonary arterial wedge pressure $(\mathrm{PAWP}) \leqslant 15 \mathrm{mmHg}$ being the current criterion for diagnosing PAH [2].

The PAWP is used as a surrogate measure of left atrial pressure, but there are limitations to its interpretation. The PAWP waveform is complex and changes during the cardiac cycle, as well as with the varying intrathoracic pressure during normal respiration. PAWP may also be affected by changes in intraalveolar pressures if the tip of the catheter rests in a region of lung where alveolar pressure intermittently exceeds pulmonary venous pressure [3]. The "gold-standard" measure of increased left heart pressure is the left ventricular end-diastolic pressure (LVEDP), which equilibrates with the pulmonary venous and left atrial pressure in diastole, except in patients with mitral valve disease or pulmonary veno-occlusive disease. It has been previously reported that in patients with $\mathrm{PH}$, the PAWP and LVEDP are frequently discordant [4]. In that series, $37 \%$ of patients with suspected PAH based on the standard PAWP-based definition had increased LVEDP (defined as $>15 \mathrm{mmHg}$ ), suggesting that $\mathrm{PAH}$ may be inappropriately diagnosed in some cases.

Some patients with left ventricular diastolic dysfunction may have normal resting LVEDP, but will show an abnormal increase in LVEDP in response to i.v. fluid loading [5, 6]. It is our routine practice to perform both right and left heart catheterisation on all patients being evaluated for $\mathrm{PH}$, and when the PAWP and LVEDP are $\leqslant 15 \mathrm{mmHg}$, the patients are given a saline load to exclude post-capillary causes of the $\mathrm{PH}$ $(\mathrm{PVH})$. Given that scleroderma is frequently associated with myocardial fibrosis and diastolic dysfunction, it is of great importance to identify these abnormalities [7-9]. Moreover, given reports of occult venoocclusive disease in some scleroderma patients [7,9], and the deleterious effects of vasodilators in this disorder, its detection is important [10]. In scleroderma, the presence of occult left ventricular diastolic dysfunction can contribute to $\mathrm{PH}$ as detected by exercise echocardiography [11]. In two previous studies of scleroderma patients undergoing haemodynamic assessment for suspected $\mathrm{PH}$, using the standard PAWPbased definition, the incidence of PVH in the PH group ranged from 33 (14\%) out of 243 to 17 (20\%) out of 83 , whereas $\mathrm{PAH}$ was diagnosed in $\sim 50 \%$ of cases $[12,13]$. The proportion of scleroderma patients suspected of having PAH but in reality having occult PVH (OPVH) is unknown. We therefore investigated the prevalence of discordance between PAWP and LVEDP in the scleroderma population, studied the frequency of OPVH as assessed by the saline challenge, and examined the epidemiological factors and echocardiographic correlates of this phenomenon.

\section{Patients and methods}

This was a retrospective analysis of all scleroderma patients referred for diagnostic right and left cardiac catheterisations at our centre between May 1, 2007 and May 31, 2011. The patients had been referred to the clinic for investigation of possible PAH due to unexplained dyspnoea, increased pulmonary artery pressure (PAP) on echocardiography $(>40 \mathrm{mmHg}$ ) or unexplained low diffusing capacity of the lung for carbon monoxide (DLCO) $(<60 \%)$. Patients with mitral valve disease on echocardiography were excluded, as were patients with evidence of chronic thromboembolic disease on lung scintigraphy. All patients underwent lung function testing and high resolution computed tomography of the lungs. Patients with decreased lung volumes (total lung capacity $<60 \%$ ), documented honeycomb changes or more than very mild basilar fibrosis on high-resolution computed tomography were excluded as having scleroderma-related interstitial lung disease. For each case, data were extracted on age, sex and the presence of hypertension, diabetes or coronary disease at the first hospital visit. None of the patients were on any therapies for PH. The protocol was approved by the Research Ethics Committee of the Jewish General Hospital (reference CR12-11).

\section{Cardiac catheterisation}

All patients underwent a full haemodynamic assessment including right and left cardiac catheterisation and coronary angiography. Left heart catheterisation was performed via a radial or femoral arterial route with a pig-tail catheter via a $6 \mathrm{~F}$ sheath. Right heart catheterisation was via the femoral route with an $8 \mathrm{~F}$ sheath able to accommodate both the Swan-Ganz catheter and the fluid infusion. Right atrial pressure, PAP and PAWP were recorded over several respiratory and cardiac cycles and measured manually as the mean of the waveform at end-expiration [14] from a single respiratory cycle after three consecutive cycles varied below 
$1 \mathrm{mmHg}$. LVEDP was measured at the onset of the electrocardiographic QRS complex. Cardiac output was determined by the thermodilution technique, with triplicate measurements. Pulmonary vascular resistance was calculated in Wood units as (mean PAP-mean PAWP)/cardiac output. Patients with resting $\mathrm{PH}$ (defined as mean PAP $>25 \mathrm{mmHg}$ ), but with low PAWP and LVEDP ( $\leqslant 15 \mathrm{mmHg}$ ) were then given an infusion of $500 \mathrm{~mL}$ of pre-warmed $0.9 \%$ saline solution over $5-10 \mathrm{~min}$, followed by remeasurement of all right and left heart haemodynamic parameters after the end of the infusion. An increase of mean PAWP or LVEDP to $>15 \mathrm{mmHg}$ was considered to be a response indicative of OPVH.

\section{Haemodynamic diagnostic algorithm}

Haemodynamic data were analysed according to the diagnostic algorithm shown in figure 1 . Patients were allocated into one of four possible diagnoses, as follows: normal (mean PAP $<25 \mathrm{mmHg}$ ); PVH; OPVH; and PAH. Diagnosis of OPVH was made when a patient with resting PAWP $\leqslant 15 \mathrm{mmHg}$ had a resting LVEDP $>15 \mathrm{mmHg}$ or a post-fluid challenge PAWP or LVEDP $>15 \mathrm{mmHg}$. Those patients did not have $\mathrm{PAH}$ and were not subsequently treated with $\mathrm{PAH}$-specific therapies.

\section{Echocardiography}

All patients underwent a comprehensive transthoracic two-dimensional and Doppler echocardiogram that was reviewed by level 3-trained echocardiographers, according to established methods [15, 16]. Data were extracted only if the echocardiogram was performed within 6 months of the catheterisation. Left atrial dimension (parasternal long axis view at end-systole) is a reflection of chronic left atrial pressure, and of the left ventricular filling pressure in the absence of mitral disease. The early transmitral filling (E) to early diastolic mitral annular velocity $\left(\mathrm{e}^{\prime}\right)\left(\mathrm{E} / \mathrm{e}^{\prime}\right)$ ratio was extracted, as this parameter is the most reliable echocardiographic index of LVEDP in haemodynamically stable patients in sinus rhythm, and has been shown to be a good discriminator between PAH and PVH $[17,18]$. The ratio between early (E) and late (atrial; A) filling was also extracted. Left ventricular systolic function was assessed as the left ventricular ejection fraction. Right ventricular function was assessed using the myocardial performance index [16]. The right ventricular systolic pressure, a surrogate for pulmonary artery systolic pressure, was estimated from the tricuspid jet velocity and an estimation of right atrial pressure according to established guidelines [16].

\section{Statistical analysis}

All continuous data were summarised as mean \pm SD, and count data were summarised as percentages. Betweengroups differences were evaluated with ANOVA (with post hoc Tukey test) or Chi-squared test as appropriate. It is noteworthy that several haemodynamic parameters (table 1) are either derived from other data in the table (e.g. pulmonary vascular resistance) or form part of the a priori definition of patient populations (mean PAP, mean PAWP and LVEDP). Between groups differences were, therefore, expected in these parameters and $\mathrm{p}$-values are reported for completeness only. The principal comparisons of interest were in mean right atrial pressure, mean PAP and carbon monoxide for haemodynamic data, and LVEF, left atrium dimension and the E/e' ratio for echocardiography. Differences between mean PAWP and LVEDP were evaluated with the BlandAltman technique. The two-sided level of significance was set at $\mathrm{p}<0.05$. The data were analysed using the $\mathrm{R}$ statistical system (version 2.11.1; The R Project for Statistical Computing, www.r-project.org).

\section{Results}

Classification into groups and demographics

We evaluated 107 scleroderma patients ( $85 \%$ females, mean age 62 years) during the study period (fig. 1). At catheterisation, 53 (50\%) patients had PH. Of these patients, based on the PAWP-based definition, 29 had PAH and 24 PVH. 11 PAH cases were reclassified as OPVH after measurement of LVEDP without $(n=5)$ or with $(n=6)$ saline challenge (fig. 1). In the PH cohort, the final prevalence of PAH was 18 (34\%) out of 53 and PVH/OPVH 35 (66\%) out of 53. With regard to demographic and laboratory data (table 2), the groups were similar for sex, age, body surface area, subtype of scleroderma and comorbidities, including systemic hypertension and diabetes. Although diuretic use tended to be higher in OPVH, the difference was not significant. Functional class differed between the groups, with normals having more patients in World Health Organization (WHO) class I and none in WHO class IV. There were no differences in haemoglobin or N-terminal pro-brain natriuretic peptide levels, but the serum creatinine was lower in normals than in $\mathrm{PVH}$ and OPVH, as were the forced vital capacity (FVC), forced expiratory volume in $1 \mathrm{~s}$ and DLCO on pulmonary function testing. Furthermore, the patients with PAH had lower levels of DLCO and higher levels of FVC/DLCO, and the OPVH group was closest to the PAH group with regard to these respiratory parameters.

\section{Haemodynamic data}

Patients classified as haemodynamically "normal" were significantly different from the PAH patients in all haemodynamic parameters except PAWP (table 1). PVH and OPVH patients were haemodynamically 


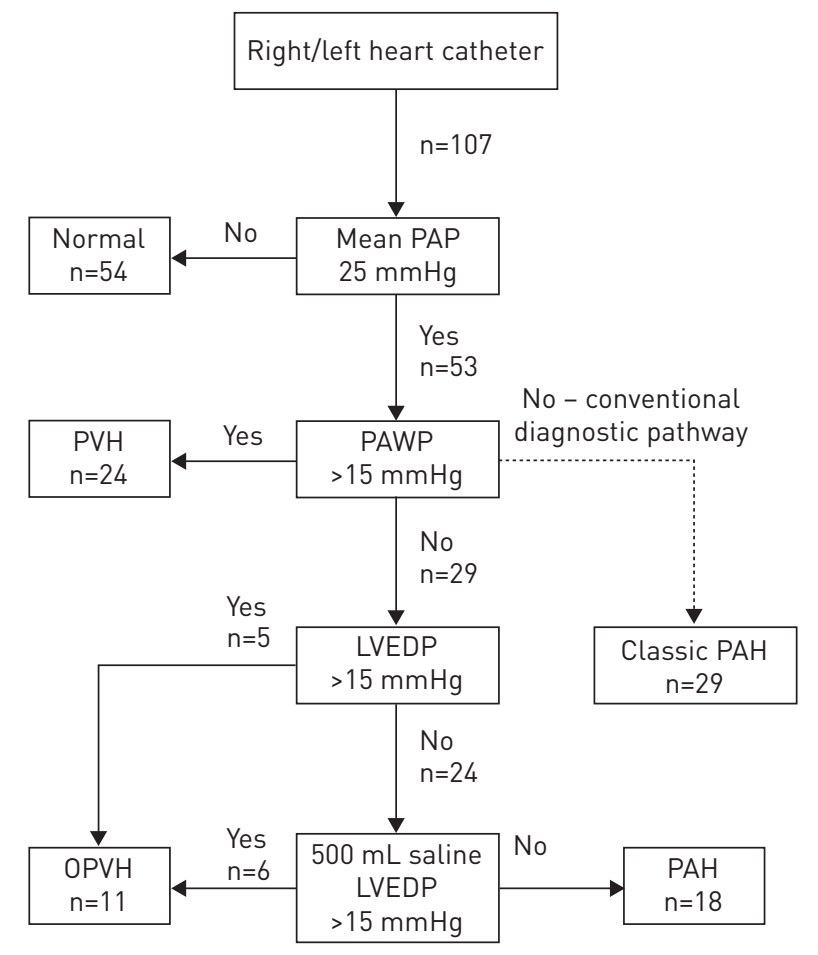

FIGURE 1 Diagnostic flowchart for patients undergoing diagnostic right and left heart catheterisation. LVEDP: left ventricular enddiastolic pressure; PAP: pulmonary artery pressure; OPVH: occult pulmonary venous hypertension; PAWP: pulmonary artery wedge pressure; $\mathrm{PAH}$ : pulmonary arterial hypertension; PVH: pulmonary venous hypertension.

similar except for mean PAWP pre-saline. PAH patients had significantly lower cardiac output and cardiac index than PVH and OPVH patients and their mean right atrial pressure was lower than that of $\mathrm{PVH}$, while their mean PAP was slightly higher than OPVH patients. Patients with PAH had significantly higher diastolic PAP-PAWP gradients (DWG), and total pulmonary gradients (TPG). No significant differences were seen in the prevalence of coronary disease.

Following fluid challenge, patients with OPVH had significantly higher LVEDP than the PAH group, which is in agreement with our definition used to differentiate the groups. When looking at the within-subject change $(\Delta)$ in pressures, the OPVH patients demonstrated significantly larger increases in LVEDP compared with the PAH patients, but not in right atrial pressure, mean PAP, or change in TPG or DWG.

In the entire dataset, the mean difference between baseline PAWP and LVEDP was $-1.3 \mathrm{mmHg}$ (95\% limits of agreement $-11.0-8.5 \mathrm{mmHg}$ ), i.e. PAWP slightly underestimates LVEDP. In the PH subgroup, the mean difference was $-0.2 \mathrm{mmHg}$ (95\% limits of agreement $-9.2-8.9 \mathrm{mmHg}$ ). It is noteworthy that, among the six patients whose LVEDP increased above $15 \mathrm{mmHg}$ after fluid challenge, the wedge pressure did not increase above $15 \mathrm{mmHg}$ in two patients (33\%).

Assuming that LVEDP $\leqslant 15 \mathrm{mmHg}$ is the gold-standard definition of pre-capillary $\mathrm{PH}$, we found that PAWP $\leqslant 15 \mathrm{mmHg}$ had sensitivity 1.0 (95\% CI $0.74-1.0$ ) and specificity 0.69 (95\% CI $0.51-0.83$ ). We performed a receiver operating characteristic analysis, which defined the optimal cut-off for PAWP $\leqslant 11 \mathrm{mmHg}$, sensitivity 0.97 (95\% CI $0.86-1.0)$ and specificity $0.78(0.55-0.91)$ with area under the curve 0.95 (95\% CI 0.89-0.99).

\section{Echocardiographic data}

In $95(89 \%)$ of the 107 patients, a transthoracic echocardiogram was performed within a median of 27 days (interquartile range 9-54 days) of the cardiac catheterisation (table 3). Systolic left ventricular ejection fraction was not different between study groups. In all echocardiographic parameters, no significant differences were demonstrated between the PVH and OPVH groups. Patients with proven PAH had a smaller left atrium dimension, higher right ventricular myocardial performance index and right ventricular tissue Doppler velocity, as well as a higher estimated systolic PAP than normal subjects, or PVH or OPVH patients. Also, there was a trend to lower $\mathrm{E} / \mathrm{e}^{\prime}$ in $\mathrm{PAH}$ patients compared with $\mathrm{PVH}$ or OPVH patients.

\section{Discussion}

The main finding of our study was that in a scleroderma population being evaluated to diagnose $\mathrm{PH}$, there was a low incidence of PAH compared with PVH, especially when OPVH cases were identified by means of 


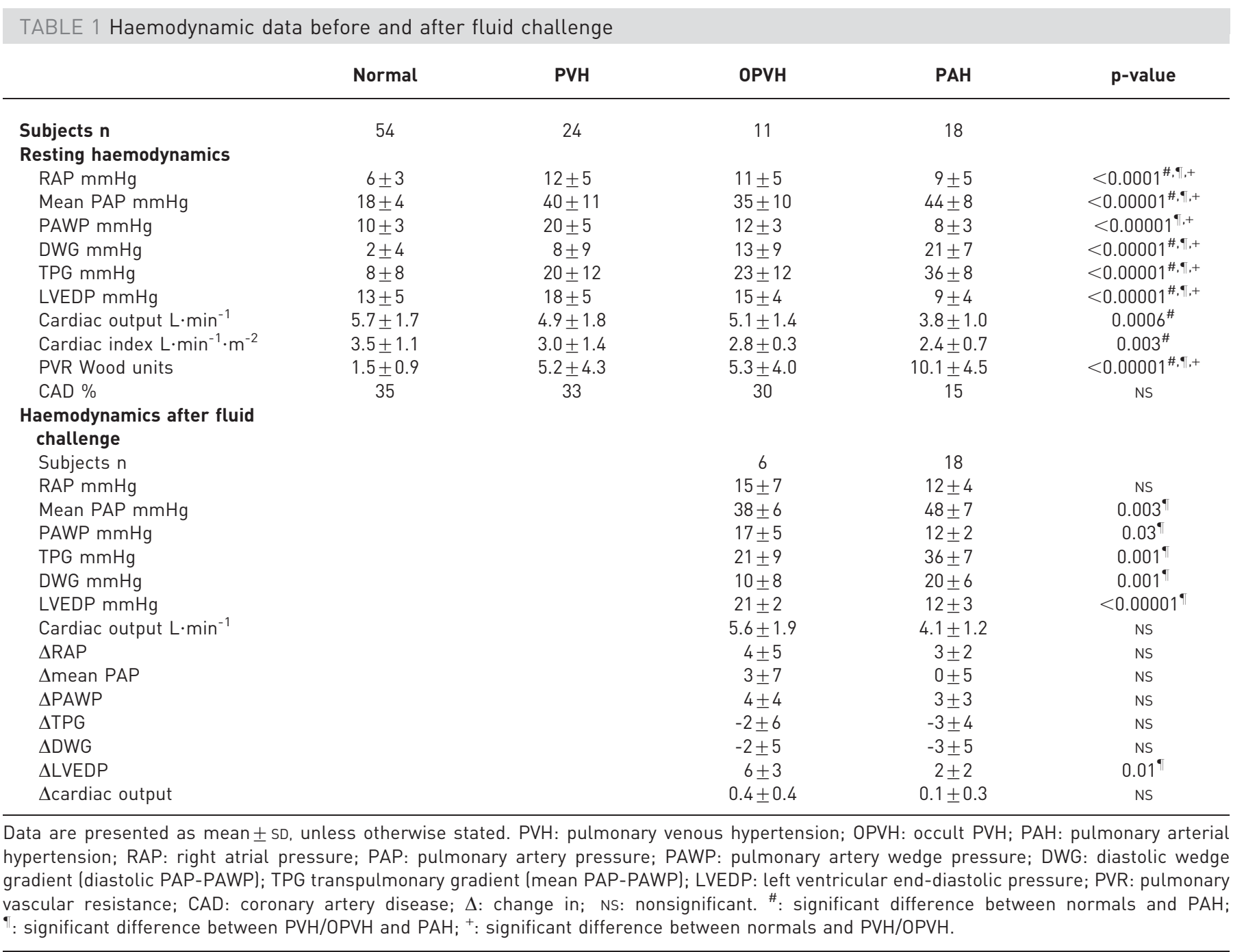

LVEDP measurements before and after fluid challenge. Using LVEDP as the gold-standard arbiter of left heart disease is appropriate from the physiological stand-point. As far as we know, this study is the first study of its kind in scleroderma patients. Another interesting observation was that, despite there being a good clinical indication for haemodynamic evaluation of the patients, only $50 \%$ had $\mathrm{PH}$ at catheterisation, as defined by current criteria. The rate of detection of true $\mathrm{PH}$ at catheterisation has varied in other scleroderma populations, and this highlights the need for better catheterisation-referral algorithms, to avoid unnecessary procedures $[12,13]$.

This study is important, as invasive haemodynamic assessment is the cornerstone of PH diagnosis. In our cohort, 29 (55\%) out of 53 scleroderma patients with $\mathrm{PH}$ had haemodynamically confirmed scleroderma$\mathrm{PAH}$ by the conventional definition, which is similar to previous reports, $[12,13]$. However, $38 \%$ of our patients had OPVH, as defined in our study. This potential misclassification of PVH as PAH may in part explain the relatively poor outcomes of scleroderma-associated PAH patients in clinical trials of PAH therapy, as these medications may vasodilate pre-capillary arteries allowing more flow through the lungs and thereby worsening left heart failure. At best, this phenomenon might explain some of the "nonresponders" to PAH therapy in those studies. Without extensive pathological data, we cannot be certain that patients whom we defined as "OPVH" are not simply a subset of scleroderma patients with genuine PAH and concomitant diastolic dysfunction. Given the high prevalence of both diseases in scleroderma patients, it would not be surprising to find them co-existing. The DLCO and FVC/DLCO data for OVPH more closely mirrored the PAH population than the PVH population, suggesting similarity at least in terms of lung disease. However, $\mathrm{PH}$ is classified and treated according to pulmonary haemodynamics rather than pathology. Therefore, we believe that defining this subpopulation is important and relevant. 
TABLE 2 Demographic and laboratory data

\begin{tabular}{|c|c|c|c|c|c|}
\hline & Normal & PVH & OPVH & PAH & p-value \\
\hline Subjects $n$ & 54 & 24 & 11 & 18 & \\
\hline Females & 85 & 88 & 64 & 94 & NS \\
\hline Age years & $61 \pm 9$ & $64 \pm 9$ & $66 \pm 6$ & $59 \pm 14$ & NS \\
\hline BSA $\mathrm{m}^{2}$ & $1.66 \pm 0.15$ & $1.68 \pm 0.21$ & $1.80 \pm 0.11$ & $1.63 \pm 0.19$ & NS \\
\hline $\begin{array}{l}\text { Scleroderma subtype } \\
\text { limited/diffuse }\end{array}$ & $70 / 30$ & $83 / 17$ & $55 / 45$ & $72 / 28$ & NS \\
\hline Hypertension & 38 & 47 & 27 & 45 & NS \\
\hline Diuretic & 24 & 47 & 87 & 50 & NS \\
\hline Diabetes mellitus & 5 & 20 & 0 & 0 & NS \\
\hline WHO functional class I/II/III/IV & $13 / 54 / 33 / 0$ & $13 / 4 / 75 / 8$ & $0 / 36 / 55 / 9$ & $0 / 28 / 44 / 28$ & $<0.001$ \\
\hline \multicolumn{6}{|l|}{ Baseline laboratory results } \\
\hline Haemoglobin $\mathrm{g} \cdot \mathrm{dL}^{-1}$ & $12.7 \pm 1.6$ & $12.5 \pm 1.6$ & $13.3 \pm 1.9$ & $12.5 \pm 1.3$ & NS \\
\hline Creatinine $\mu \mathrm{mol} \cdot \mathrm{L}^{-1}$ & $75 \pm 26$ & $95 \pm 23$ & $91 \pm 24$ & $87 \pm 39$ & $0.04^{\#}$ \\
\hline $\mathrm{NT}-$ proBNP $\mathrm{pg} \cdot \mathrm{mL}^{-1}$ & $366 \pm 750$ & $2880 \pm 1916$ & $721 \pm 899$ & $5678 \pm 9067$ & NS \\
\hline \multicolumn{6}{|l|}{ Lung function tests } \\
\hline FVC \% pred & $86 \pm 16$ & $72 \pm 17$ & $69 \pm 17$ & $73 \pm 20$ & $0.02^{\#}$ \\
\hline FEV $1 \%$ pred & $81 \pm 17$ & $69 \pm 16$ & $66 \pm 14$ & $68 \pm 29$ & $0.03^{\#, \Phi}$ \\
\hline TLC \% pred & $90 \pm 14$ & $81 \pm 17$ & $74 \pm 7$ & $82 \pm 20$ & $0.02^{\#}$ \\
\hline DLco \% pred & $61 \pm 17$ & $56 \pm 15$ & $36 \pm 7$ & $32 \pm 6$ & $<0.001^{\#, \Phi_{\text {, }}}$ \\
\hline FVC/DLCO & $1.5 \pm 0.5$ & $1.5 \pm 0.6$ & $2.0 \pm 0.6$ & $2.4 \pm 1.0$ & $0.003^{+,+}$ \\
\hline
\end{tabular}

TABLE 3 Echocardiographic parameters from patients performed after right heart catheterisation"

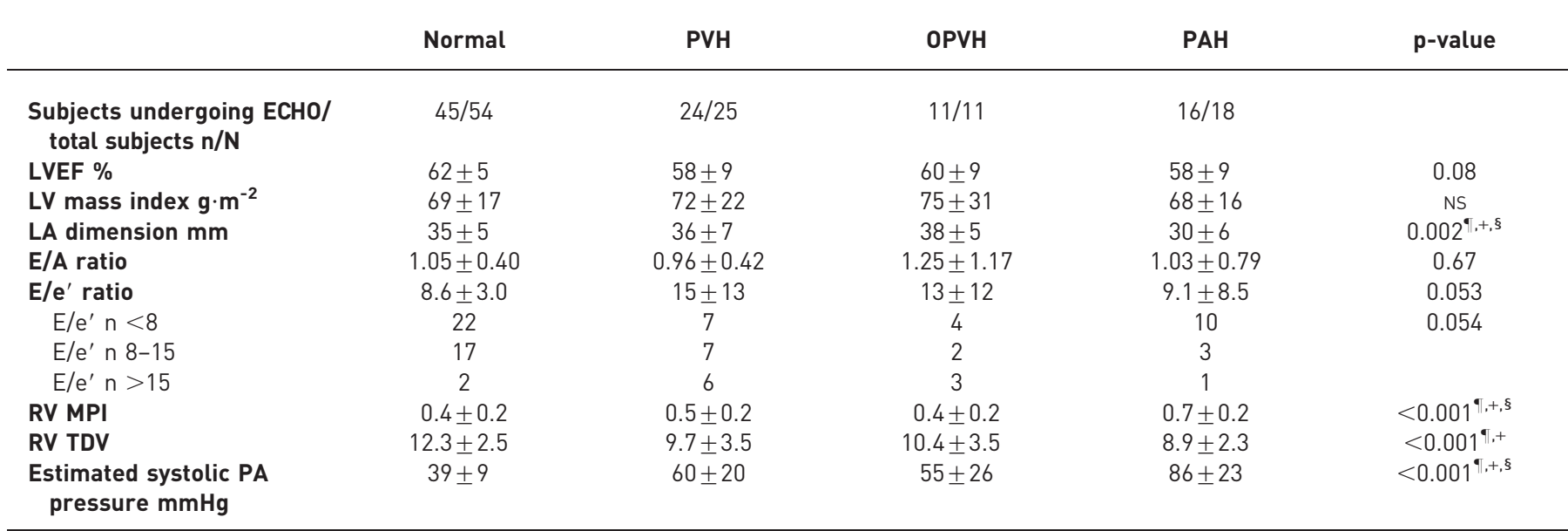

Data are presented as mean \pm SD, unless otherwise stated. PVH: pulmonary venous hypertension; OPVH: occult PVH; PAH: pulmonary arterial hypertension; ECHO: echocardiography; LVEF: left ventricular ejection fraction; LV: left ventricular; LA: left atrium; E/A: ratio between early (E) and late atrial (A) filling; E/e': the early transmitral filling (E) to early diastolic mitral annular velocity (e'); RV: right ventricular; MPI: myocardial performance index; TDV: tissue Doppler velocity; PA: pulmonary artery. " ${ }^{\text {: }}$ performed at a median of 26 days (interquartile range 9-49 days); ${ }^{\top}$ : significant difference between normals and $\mathrm{PAH}_{;}{ }^{+}$: significant difference between $\mathrm{PVH} / \mathrm{OPVH}$ and $\mathrm{PAH} ;{ }^{\S}$ : significant difference between normals and $\mathrm{PVH} / \mathrm{OPVH}$. 
Moreover, a central principle in the diagnosis of PAH is that there is no post-capillary component. Thus, the ability to identify PAH is always hampered in the presence of post-capillary disease, and this is a particularly vexing issue in the scleroderma population. Additionally, some of the patients might have had a component of occult veno-occlusive disease $[7,9,10]$. It is unknown whether pulmonary veno-occlusive disease (PVOD) might be revealed by a saline challenge, or whether the PAWP would remain low. This would require a separate study in a larger population of pre-identified patients with high-risk features for PVOD [10], a normal resting PAWP, and perhaps a more modest saline challenge in view of the risks of inducing pulmonary oedema. GÜNTHER et al. [10] have reported a significant incidence of radiological findings suggestive of PVOD, suggesting a high incidence. We did not see this in our population, but it is possible that some of the PVOD was "screened out" before patients were referred to us for evaluation. Cardiac magnetic resonance imaging might also help detect patients with left ventricular involvement by scleroderma, but we did not have access to magnetic resonance imaging for the present study.

For patients with PVH, it has been suggested in guidelines that the TPG (mean PAP-PAWP) may be helpful in distinguishing "reactive" pre-capillary pulmonary vascular remodelling from a "passive" increase in PAWP, where the TPG is $<12 \mathrm{mmHg}$ in the latter [19, 20]. The change in TPG during exercise has also been used to study the response of PAH to therapy during exercise cardiac catheterisation in scleroderma patients [21]. In our study, both the TPG and DWG were lower in PVH (occult or not) than in PAH, consistent with less pre-capillary remodelling. Furthermore, after saline challenge, the TPG and DWG remained lower in OPVH than in PAH, and the change in TPG and DWG post saline was the same in $\mathrm{OPVH}$ and PAH. This suggests that the haemodynamic impairment, caused by whatever degree of precapillary structural remodelling is present, does not change with saline infusion. It is notable that in all three pulmonary hypertensive groups, DWG and TPG were higher than normal, suggesting some degree of precapillary disease, which was, as expected, the most extreme in PAH.

An additional novel finding in our study is that several (six (25\%) out of 24) patients with low PAWP and low resting LVEDP who were re-evaluated after a modest fluid challenge were recognised as having OPVH rather than PAH. Although the need for fluid challenges to exclude $\mathrm{PVH}$ is described in the literature, there is little data regarding this diagnostic manoeuvre in $\mathrm{PH}$ patients. The effect of volume loading on LVEDP has been studied in several small physiological studies. The most frequently cited study focused on the effects of fluid challenge in patients with constrictive pericarditis, but also studied normal subjects and postmyocardial infarction patients [6]. Administration of $1 \mathrm{~L}$ of saline over 6-8 $\mathrm{min}$ in the six normal subjects raised the LVEDP/pulmonary capillary wedge pressure (PCWP) by a maximum of $3 \mathrm{mmHg}$, and LVEDP/ PCWP remained below $12 \mathrm{mmHg}$ in all cases [6]. In the same study, LVEDP/PAWP post-volume expansion was $>15 \mathrm{mmHg}$ in half of the patients who had previous myocardial infarction. Grossman et al. [22] increased preload by sudden passive elevation of the legs to a right angle to the trunk. Three of the subjects, aged 23, 50 and 55 years, had normal hearts. Measurements performed at $60 \mathrm{~s}$, which would eliminate any Valsalva effect, showed LVEDPs of 10, 12 and $6 \mathrm{mmHg}$, respectively, with a maximum rise of $2 \mathrm{mmHg}$ as compared with baseline [22]. QUINONES et al. [23] also studied the effects of acute preload increase in humans. However, their goal was to deliberately raise LVEDP to $\geqslant 15 \mathrm{mmHg}$ by rapid i.v. infusion of dextran solution and, thus, their study cannot be used to interpret the effects of a non-LVEDP target-directed fluid challenge. In a population at high risk for diastolic dysfunction associated with the metabolic syndrome, administration of only $500 \mathrm{~mL}$ saline over $5 \mathrm{~min}$ was able to reveal subjects where the PCWP increased by an average of $6.8 \mathrm{mmHg}$, to $>15 \mathrm{mmHg}$ [24]. Thus, while the data derived from volume expansion may not detect all cases of diastolic dysfunction, they seem to exclude diastolic dysfunction in normal controls. It might also be argued that the increased left-sided heart pressures seen after saline challenge represent increased filling of a dilated right heart, causing worsening ventricular interdependence. However, this hypothesis is refuted by the fact that in all cases, the rise in left heart pressure was greater than any rise in right sided pressures after the saline bolus (change in pressures; table 1). Further studies are needed to better define the "normal" LVEDP response to a standardised fluid challenge across a wide spectrum of normal subjects and patients of different age, sex and disease in order to refine the diagnostic utility of this manoeuvre.

Our study extends two previous investigations, but it is the only analysis to contain exclusively scleroderma patients and the first paper in $\mathrm{PH}$ patients in general to formally describe the possible diagnostic utility of the saline challenge. HALPERN and TAICHMAN [4] reported significant discrepancies between PAWP and LVEDP in a very large database $(n=11523)$ of right and left heart catheterisations performed in their centre. Amongst those patients with PAH according to the PAWP, 53.5\% were found to have elevated LVEDP. Sото et al. [25] reported in abstract form 131 right and left cardiac catheterisations performed in their centre and demonstrated that $37 \%$ of patients with PAH according to PAWP had OPVH according to resting LVEDP. Our estimate of $38 \%$ overdiagnosis of $\mathrm{PAH}$ is consistent with these previous estimates. In a Bland-Altman analysis performed by HALPERN and TAICHMAN [4], PAWP was found to underestimate LVEDP by $2.9 \mathrm{mmHg}$ with $95 \%$ limits of agreement of -12.9 to $+9.5 \mathrm{mmHg}$. Our analysis had similar 
results. The mean difference between PAWP and LVEDP is very small in the overall population $(-0.2 \mathrm{mmHg})$, but the limits of agreement are wide $(-9.2$ to $+8.9 \mathrm{mmHg})$, such that PAWP cannot be reliably interpreted in an individual patient without an LVEDP unless the value is very low $(<6 \mathrm{mmHg})$. Furthermore, PAWP $\leqslant 15 \mathrm{mmHg}$ lacks specificity (68\%) for detecting cases with LVEDP $\leqslant 15 \mathrm{mmHg}$, and even at the optimal threshold of PAWP $\leqslant 11 \mathrm{mmHg}$, as suggested by receiver operating characteristic analysis of the data, specificity increased to only $78 \%$.

The data presented here also extend the work of HALPERN and TAICHMAN [4] by evaluating the effect of demographic factors on final $\mathrm{PH}$ diagnosis in scleroderma patients. We did not find any parameters that could help physicians predict which scleroderma patients are at higher risk of OPVH. This could possibly be explained by the relatively small sample size, and further research is needed in this area. It is recognised that patients with the metabolic syndrome have increased incidence of $\mathrm{PVH}$, but we did not have reliable data on this factor for analysis [24].

Our study also provides important echocardiographic evidence for the similarity between OPVH and PVH patients, relative to PAH, in scleroderma patients with suspected PH. In our study, none of the patients had haemodynamically significant valvular abnormalities. Although the systolic function was preserved across the entire patient cohort, we demonstrated that patients with PVH or OPVH had evidence of increased filling pressures on echocardiography (higher E/e') [11]. This is consistent with a previous study comparing echocardiographic parameters in patients with proven idiopathic PAH versus $\mathrm{PVH}$, where E/e' had $97 \%$ area under the curve for differentiating between the two conditions [13]. Furthermore, the left ventricular mass index was not able to distinguish between the groups. Patients with PAH have relatively small left atrial dimensions, which is consistent with an underfilled left heart and thus lower left-sided pressures. We do not suggest that echocardiography can substitute a full invasive haemodynamic assessment.

Our study has all the limitations associated with being a single-centre retrospective analysis. Despite that, the saline challenge was performed systematically, which reduces the possibility of selection bias within our population. Of course, referral bias might still be present, in that the scleroderma patients were referred to us for catheterisation only because of dyspnoea, a fall in carbon monoxide diffusing capacity in the absence of lung fibrosis, or an echocardiogram suggesting PH. Thus we cannot state what the prevalence of OPVH is in a general scleroderma population that has no present indication for catheterisation. However, because we systematically performed left heart catheterisations once there was an indication for catheterisation, the percentage of $\mathrm{PVH}$ in a population with an indication should not reflect selection bias. We did find a higher percentage of PVH than was previously described by SCHREIBER et al. [12] and AvOUAC et al. [13]. We do not have an explanation for this, although it is increasingly recognised that left heart disease is highly prevalent in North American populations.

In summary, a complete invasive haemodynamic assessment, including measurement of LVEDP with an appropriate saline challenge in scleroderma patients being evaluated for $\mathrm{PH}$, may be critical to making the correct haemodynamic diagnosis by revealing a subset of patients with OPVH. However, further detailed clinical study is required to determine whether the clinical course and outcomes of the OPVH group are different from those of PAH, and whether the presence of OPVH has any impact on therapeutic success.

\section{Acknowledgements}

We thank the staff of the cardiac catheterisation laboratory for their generous assistance, and their sympathetic care of the patients.

\section{References}

1 Steen VD, Lucas M, Fertig N, et al. Pulmonary arterial hypertension and severe pulmonary fibrosis in systemic sclerosis patients with a nucleolar antibody. J Rheumatol 2007; 34: 2230-2235.

2 McLaughlin VV, Archer SL, Badesch DB, et al. ACCF/AHA 2009 expert consensus document on pulmonary hypertension: a report of the American College of Cardiology Foundation Task Force on Expert Consensus Documents and the American Heart Association: developed in collaboration with the American College of Chest Physicians, American Thoracic Society, Inc., and the Pulmonary Hypertension Association. Circulation 2009; 119: 2250-2294.

3 West JB. Respiratory Physiology - The Essentials. 6th Edn. Philadelphia, Lippincott Williams \& Wilkins, 2000.

4 Halpern SD, Taichman DB. Misclassification of pulmonary hypertension due to reliance on pulmonary capillary wedge pressure rather than left ventricular end-diastolic pressure. Chest 2009; 136: 37-43.

5 Hemnes AR, Forfia PR, Champion HC. Assessment of pulmonary vasculature and right heart by invasive haemodynamics and echocardiography. Int J Clin Pract Suppl 2009; 162: 4-19.

6 Bush CA, Stang JM, Wooley CF, et al. Occult constrictive pericardial disease. Diagnosis by rapid volume expansion and correction by pericardiectomy. Circulation 1977; 56: 924-930.

7 Johnson SR, Patsios D, Hwang DM, et al. Pulmonary veno-occlusive disease and scleroderma associated pulmonary hypertension. J Rheumatol 2006; 33: 2347-2350.

8 Desai CS, Lee DC, Shah SJ. Systemic sclerosis and the heart: current diagnosis and management. Curr Opin Rheumatol 2011; 23: 545-554. 
Dorfmüller P, Montani D, Humbert M. Beyond arterial remodelling: pulmonary venous and cardiac involvement in patients with systemic sclerosis-associated pulmonary arterial hypertension. Eur Respir J 2010; 35: 6-8.

10 Günther S, Jaïs X, Maitre S, et al. Computed tomography findings of pulmonary venoocclusive disease in scleroderma patients presenting with precapillary pulmonary hypertension. Arthritis Rheum 2012; 64: 2995-3005.

11 D'Alto M, Ghio S, D'Andrea A, et al. Inappropriate exercise-induced increase in pulmonary artery pressure in patients with systemic sclerosis. Heart 2011; 97: 112-117.

12 Schreiber BE, Valerio CJ, Keir GJ, et al. Improving the detection of pulmonary hypertension in systemic sclerosis using pulmonary function tests. Arthritis Rheum 2011; 63: 3531-3539.

13 Avouac J, Airò P, Meune C, et al. Prevalence of pulmonary hypertension in systemic sclerosis in European Caucasians and metaanalysis of 5 studies. J Rheumatol 2010; 37: 2290-2298.

14 Ryan JJ, Rich JD, Thiruvoipati T, et al. Current practice for determining pulmonary capillary wedge pressure predisposes to serious errors in the classification of patients with pulmonary hypertension. Am Heart J 2012; 163: 589-594.

15 Lang RM, Bierig M, Devereux RB, et al. Recommendations for chamber quantification: a report from the American Society of Echocardiography's guidelines and standards committee and the chamber quantification writing group, developed in conjunction with the European Association of Echocardiography, a branch of the European Society of Cardiology. J Am Soc Echocardiogr 2005; 18: 1440-1463.

16 Rudski LG, Lai WW, Afilalo J, et al. Guidelines for the echocardiographic assessment of the right heart in adults: a report from the American Society of Echocardiography endorsed by the European Association of Echocardiography, a registered branch of the European Society of Cardiology, and the Canadian Society of Echocardiography. J Am Soc Echocardiogr 2010; 23: 685-713.

17 Park JH, Marwick TH. Use and limitations of E/e' to assess left ventricular filling pressure by echocardiography. J Cardiovasc Ultrasound 2011; 19: 169-173.

18 Willens HJ, Chirinos JA, Gomez-Marin O, et al. Noninvasive differentiation of pulmonary arterial and venous hypertension using conventional and Doppler tissue imaging echocardiography. J Am Soc Echocardiogr 2008; 21: 715-719.

19 Paulus WJ, Tschöpe C, Sanderson JE, et al. How to diagnose diastolic heart failure: a consensus statement on the diagnosis of heart failure with normal left ventricular ejection fraction by the Heart Failure and Echocardiography Associations of the European Society of Cardiology. Eur Heart J 2007; 28: 2539-2550.

20 Hoeper MM, Barberà JA, Channick RN, et al. Diagnosis, assessment, and treatment of non-pulmonary arterial hypertension pulmonary hypertension. J Am Coll Cardiol 2009; 54: Suppl. 1, S85-S96.

21 Saggar R, Khanna D, Shapiro S, et al. Brief report: effect of ambrisentan on exrecise-induced pulmonary hypertension in systemic sclerosis: a prospective single-center, open-label study. Arthritis Rheum 2012; 64: 4072-4077.

22 Grossman W, Haynes F, Paraskos JA, et al. Alterations in preload and myocardial mechanics in the dog and in man. Circ Res 1972; 31: 83-94.

23 Quinones MA, Gaasch WH, Alexander JK. Influence of acute changes in preload, afterload, contractile state and heart rate on ejection and isovolumic indices of myocardial contractility in man. Circulation 1976; 53: 293-302.

24 Robbins IM, Newman JH, Johnson RF, et al. Association of the metabolic syndrome with pulmonary venous hypertension. Chest 2009; 136: 31-36.

25 Soto FJ, Siegel R, Marks D, et al. Performance of pulmonary capillary wedge pressure (PCWP) vs. left ventricular end diastolic pressure (LVEDP) in the diagnosis/classification of patients with suspected pulmonary arterial hypertension (PAH). Chest 2005; 128: 137S. 